\title{
Additional important research priorities for bronchiectasis in China
}

\author{
To the Editor:
}

We highly appreciate the laudable desire of the European Multicentre Bronchiectasis Audit and Research Collaboration (EMBARC) [1] to provoke research interests and develop study protocols to investigate bronchiectasis, a disease which has not yet received sufficient attention worldwide, particularly in China. China is a major developing country with a large population; however, there is a lack of epidemiological investigations detailing the prevalence or incidence of bronchiectasis. While we totally concur with the recommendations from European experts, we feel that additional priorities in bronchiectasis should be highlighted for future research in China.

1) Regional distribution of bronchiectasis aetiology throughout China. The aetiological investigations published to date were mainly based in major cities such as Shanghai [2], Guangzhou [3], and Jinan [4]. The prevalence of pulmonary infections (e.g. tuberculosis, measles and pertussis) are expected to be significantly higher in rural areas because of the limited medical resources. Our understanding has been hampered by the lack of official documentation regarding regional distributions of bronchiectasis aetiologies in China. Future investigations are warranted to prioritise our national healthcare plan for disease control.

2) The role of traditional Chinese medicine in bronchiectasis patients. The long Chinese history has nurtured the development of traditional Chinese medicine, a delicate medical system which is still regarded as a tremendous treasure trove that is complementary to western medicine. In fact, a considerable number of bronchiectasis patients report that they have actively sought consultation from experts in traditional Chinese medicine. Patients had greater willingness to receive therapy with Chinese herbal compounds (typically for decoction) to minimise the potential adverse effects of western medicine (W.J. Guan and co-workers; unpublished data). To our knowledge, the efficacy of Chinese herbs for improving asthma control has been documented [5]. We strongly feel that Chinese herbs may have a role in future management of bronchiectasis, for instance, by reducing the frequency of bronchiectasis exacerbations. However, in light of the lack of evidence-based reports, well-designed randomised, double-blind clinical trials are needed to thoroughly validate the efficacy and safety of Chinese herbs for bronchiectasis.

3) The role of clinical phenotyping in improving management of bronchiectasis. The significant heterogeneity of clinical manifestations of bronchiectasis has been increasingly recognised. Clinical phenotyping using unsupervised learning techniques $[6,7]$ has provided researchers with an invaluable opportunity to personalise therapy. Despite the absence of "gold standard criteria" for classification of clusters, clinical phenotyping is complementary to disease severity assessment because it takes into account both the homogeneity and heterogeneity of bronchiectasis. To justify bronchiectasis phenotyping, it would be useful to investigate its added value to improve therapeutic outcomes and long-term prognosis.

4) The impact of bronchiectasis on fertility. Compared with Caucasians [6, 8, 9], Chinese bronchiectasis patients are significantly younger at enrolment [2-4, 7]. The mean age of our cohort was 44.5 years [6], and $\sim 62 \%$ of females were still of child-bearing age. Currently, no report documenting whether bronchiectasis decreased fertility has been published. Nonetheless, clinicians have been frequently confronted with fertility counselling for bronchiectasis patients. It has become increasingly urgent to solve this issue after the implementation of the two-child policy in China [10]. In our pilot study (W.J. Guan and co-workers; unpublished data), most female patients demonstrated varying magnitudes of concern about infertility associated with bronchiectasis, despite the fact that most females successfully gave birth to healthy infants. Concerns about infertility have cast a considerable shadow over patient's daily life, leading to the development of anxiety and/or depression. Addressing the impact of bronchiectasis on fertility may

@ERSpublications

Aetiology, Chinese medicine, phenotyping, fertility and comorbidities of bronchiectasis should be studied in China http://ow.ly/tiJl304s5j0

Cite this article as: Guan W-jie, Gao Y-hua, Yuan J-jing, et al. Additional important research priorities for bronchiectasis in China. Eur Respir J 2017; 49: 1601747 [https://doi.org/10.1183/13993003.01747-2016]. 
help advance future healthcare in terms of adding further evidence to genetic counselling and mitigating patient's anxiety or depression.

5) Pathophysiological pathways leading to bronchiectasis-chronic obstructive pulmonary disease (COPD) and bronchiectasis-asthma overlap syndrome. There are various aetiologies underlying the pathogenesis of bronchiectasis. In the British Thoracic Society guidelines [11], COPD or asthma should be considered as the underlying aetiology of bronchiectasis. It should also be recognised that the determination of aetiology is challenging in most patients, but the overlap of bronchiectasis with asthma or COPD can be assessed using simple tools such as lung function tests. Current knowledge indicates that bronchiectasis may have common pathways with asthma (e.g. eosinophilic and/or neutrophilic inflammation) and COPD (e.g. neutrophilic and/ or macrophagic inflammation). Bronchiectasis can be readily identified in a considerable number of COPD and asthmatic patients using high-resolution chest computed tomography, particularly in those with a prolonged course of disease. How primary or secondary aetiologies stimulate exaggerated neutrophilic responses leading to relentless degradation of the matrices of bronchial mucosa remains an area of profound interest. Unravelling the most useful biomarkers (e.g. periostin or fractional exhaled nitric oxide) to classify the clinical clusters and predict prognosis would provide clinicians with practical tools for disease intervention and management.

These additional research priorities in China may have complementary significance to the statement from the EMBARC Clinical Research Collaboration [1]. Researchers from China keenly await collaboration with colleagues from western countries to collectively solve the unanswered questions associated with bronchiectasis management.

Wei-jie Guan ${ }^{1}$, Yong-hua Gao ${ }^{2}$, Jing-jing Yuan ${ }^{1}$, Rong-chang Chen ${ }^{1}$ and Nan-shan Zhong ${ }^{1}$

${ }^{1}$ State Key Laboratory of Respiratory Disease, National Clinical Research Center for Respiratory Disease, Guangzhou Institute of Respiratory Disease, First Affiliated Hospital of Guangzhou Medical University, Guangzhou Medical University, Guangzhou, China. ${ }^{2}$ Dept of Respiratory and Critical Care Medicine, First Affiliated Hospital of Zhengzhou University, Zhengzhou, China.

Correspondence: Rong-chang Chen, State Key Laboratory of Respiratory Disease, National Clinical Research Center for Respiratory Disease, Guangzhou Institute of Respiratory Disease, First Affiliated Hospital of Guangzhou Medical University, 151 Yanjiang Road, Guangzhou, Guangdong, China. E-mail: chenrc@vip.163.com

Received: Sept 012016 | Accepted: Sept 022016

Support statement: Changiiang Scholars and Innovative Research Team in University (grant number: ITR0961), the National Key Technology R\&D Program of the 12th National Five-year Development Plan (grant number: 2012BAI05B01) and National Key Scientific \& Technology Support Program: Collaborative innovation of Clinical Research for chronic obstructive pulmonary disease and lung cancer grant number 2013BAI09B09 (to N.S. Zhong and R.C. Chen), National Natural Science Foundation (grant number: 81400010), and 2014 Scientific Research Projects for Medical Doctors and Researchers from Overseas, Guangzhou Medical University grant number 2014C21 (to W.J. Guan). Funding information for this article has been deposited with the Open Funder Registry.

Conflict of interest: Disclosures can be found alongside this article at erj.ersjournals.com

Acknowledgements: Author contributions: W.J.G., Y.H.G. and J.J.Y. drafted the manuscript; R.C.C. and N.S.Z. critically reviewed the manuscript and approved final submission.

\section{References}

1 Aliberti S, Masefield S, Polverino E, et al. Research priorities in bronchiectasis: a consensus statement from the EMBARC Clinical Research Collaboration. Eur Respir J 2016; 48: 632-647.

2 Mao B, Yang JW, Lu HW, et al. Asthma and bronchiectasis exacerbation. Eur Respir J 2016; 47: 1680-1686.

3 Guan WJ, Gao YH, Xu G, et al. Aetiology of bronchiectasis in Guangzhou, southern China. Respirology 2015; 20: 739-748.

4 Qi Q, Wang W, Li T, et al. Aetiology and clinical characteristics of patients with bronchiectasis in a Chinese Han population: a prospective study. Respirology 2015; 20: 917-924.

5 Wen $\mathrm{MC}$, Wei $\mathrm{CH}, \mathrm{Hu} \mathrm{ZQ}$, et al. Efficacy and tolerability of anti-asthma herbal medicine intervention in adult patients with moderate-severe allergic asthma. J Allergy Clin Immunol 2005; 116: 517-524.

6 Aliberti S, Lonni S, Dore S, et al. Clinical phenotypes in adult patients with bronchiectasis. Eur Respir J 2016; 47: $1113-1122$.

7 Guan WJ, Jiang $\mathrm{M}$, Gao $\mathrm{YH}$, et al. Unsupervised learning technique identifies bronchiectasis phenotypes with distinct clinical characteristics. Int J Tuberc Lung Dis 2016; 20: 402-410.

8 McDonnell MJ, Aliberti S, Goeminne PC, et al. Multidimensional severity assessment in bronchiectasis: an analysis of seven European cohorts. Thorax 2016; in press [DOI: 10.1136/thoraxjnl-2016-208481].

9 Chalmers JD, Smith MP, McHugh BJ, et al. Short- and long-term antibiotic treatment reduces airway and systemic inflammation in non-cystic fibrosis bronchiectasis. Am J Respir Crit Care Med 2012; 186: 657-665.

10 Sina English. Two-child policy is a turning point for China. http://english.sina.com/china/2015/1030/860727.html Date last accessed: September 28, 2016. Date last updated: October 31, 2015.

11 Pasteur MC, Bilton D, Hill AT et al. British Thoracic Society guideline for non-CF bronchiectasis. Thorax 2010; 65: Suppl., i1-i58. 
From the authors:

We thank W. Guan and colleagues for highlighting important additional research priorities for bronchiectasis in China. The European Multicentre Bronchiectasis Audit and Research Collaboration (EMBARC) research priorities "roadmap" published in the European Respiratory Journal was specifically developed to address the issue of research priorities from the point of view of European experts, and with this in mind, it is important to consider how these priorities may be different across different healthcare systems in differing areas of the world [1].

Bronchiectasis appears to be heterogeneous across the world. In addition to the different characteristics observed in China compared to Europe, the emerging Indian Bronchiectasis Registry, as part of the EMBARC network, has reported a high rate of post-tuberculosis bronchiectasis and bronchiectasis related to chronic obstructive pulmonary disease $[2,3]$. A further research priority is therefore to understand the prevalence, impact and outcomes of bronchiectasis following tuberculosis infection.

In the US Bronchiectasis Research Registry, 63.4\% of patients had a history of pulmonary nontuberculous mycobacteria (NTM) being present, an association, aetiology or complication seen much less frequently in Europe [4]. We may therefore add from a North American perspective, a key priority to identify the optimal treatment for NTM infections as well as understanding the susceptibility factors and disease characteristics associated with NTM infection with or without bronchiectasis [5]. While many of the research priorities for colleagues in Australasia would be similar to those of Europe, we might add priorities to understanding the burden of disease and aetiology in indigenous populations [6].

Therefore, it is clear that across the world, patient populations and therefore research priorities may be different yet complementary, representing a tremendous opportunity to further inform the scientific community and advance the science of bronchiectasis. Nevertheless, the desire and well overdue need to improve patient outcomes and to find evidence for new treatments remains the same [7].

It is in this spirit that the international collaborative framework for the investigation bronchiectasis is now well established. The EMBARC network (www.bronchiectasis.eu) is closely aligned with research efforts in many individual European countries, but most importantly is collaborating closely with international bronchiectasis networks [8]. The US Bronchiectasis Research Registry began data collection in 2008 across 13 centres and incorporates data collection for bronchiectasis as well as NTM. The Australian Bronchiectasis Registry, under the auspices of the Australian Lung Foundation, has recently begun recruitment and has the unique feature of collecting data on children. EMBARC India, the Indian bronchiectasis registry, is far advanced with $>500$ patients enrolled at the time of writing from $>33$ centres. Emerging bronchiectasis research programmes are in development in other countries, including in Latin America, Sri Lanka and South Africa.

An important symbol of this new international consortium of bronchiectasis researchers was the first World Bronchiectasis Conference (WBC) in Hannover, Germany, in July 2016 (http://world-bronchiectasisconference.com). This event saw the participation of $>300$ delegates from around the world who met to discuss clinical and translational aspects of bronchiectasis under the auspices of the European Respiratory Society, EMBARC and PROGNOSIS, and in collaboration with the US Bronchiectasis Research Registry and Australian Bronchiectasis Registry. In the wake of this major success, the second WBC has been organised to take place in Milan, Italy, in July 2017, with a special focus of bronchiectasis clinical trials and pulmonary NTM.

We invite all those with an interest in bronchiectasis to participate in these international collaborative efforts. Specific objectives of our collaborations are: 1) to merge different bronchiectasis registries on a common platform with shared definitions to allow comparative global studies and generalisable and well as unique research findings; 2) to identify, integrate and compare research needs in the field; 3 ) to share infrastructures and competencies in order to design and conduct high-quality clinical intervention trials according to a shared roadmap; 4) to plan educational policies to increase disease awareness, and expand the clinical and scientific communities interested in bronchiectasis [9]; and 5) to facilitate the interaction between international patients with bronchiectasis. This integrated and multinational approach, we believe,

@ERSpublications

A new international consortium of bronchiectasis researchers has been launched http://ow.ly/HfK4307adkV

Cite this article as: Aliberti S, Dhar R, Aksamit TR, et al. Additional important research priorities for bronchiectasis in China. Eur Respir J 2017; 49: 1602317 [https://doi.org/10.1183/13993003.02317-2016]. 
represents a powerful and effective pathway to achieve progress not just in one part of the world, but for the bronchiectasis globally.

We look forward to working with our international colleagues who share the core priorities to improve the understanding of, quality of life of, treatment options for and outcomes of patients with bronchiectasis.

Stefano Aliberti $\odot^{1}$, Raja Dhar ${ }^{2}$, Timothy R. Aksamit ${ }^{3}$, Lucy Morgan ${ }^{4}$ and James D. Chalmers ${ }^{5}$

${ }^{1}$ Dept of Pathophysiology and Transplantation, University of Milan, Cardio-thoracic unit and Adult Cystic Fibrosis Center, Fondazione IRCCS Cà Granda Ospedale Maggiore Policlinico, Milan, Italy. ${ }^{2}$ Fortis Hospital, Pulmonology and Critical Care, Kolkata, India. ${ }^{3}$ Mayo Clinic, Pulmonary Disease and Critical Care Medicine, Rochester, MN, USA. ${ }^{4}$ Department of Respiratory Medicine, Concord Hospital, Concord Clinical School, University of Sydney, Sydney, Australia. ${ }^{5}$ Tayside Respiratory Research Group, University of Dundee, Dundee, UK.

Correspondence: Stefano Aliberti, Dept of Pathophysiology and Transplantation, University of Milan, Cardio-thoracic unit and Cystic Fibrosis Adult Center, Fondazione IRCCS Ca' Granda Ospedale Maggiore Policlinico, Via Francesco Sforza 35, 20122, Milan, Italy. E-mail: stefano.aliberti@unimi.it

Received: Nov 252016 | Accepted: Nov 252016

Conflict of interest: Disclosures can be found alongside this article at erj.ersjournals.com

\section{References}

1 Aliberti S, Masefield S, Polverino E, et al. Research priorities in bronchiectasis: a consensus statement from the EMBARC Clinical Research Collaboration. Eur Respir J 2016; 48: 632-637.

2 Lonni S, Chalmers JD, Goeminne PC, et al. Etiology of non-cystic fibrosis bronchiectasis in adults and its relationship to severity. Ann Am Thorac Soc 2015; 12: 1764-1770.

3 Mao B, Yang JW, Lu HW, et al. Asthma and bronchiectasis exacerbation. Eur Respir J 2016; 47: 1680-1686.

4 Aksamit TR, Carretta E, Daley CL, et al. The Bronchiectasis Research Registry: a collaborative research cohort for non-cystic fibrosis bronchiectasis. Am J Respir Crit Care Med 2012; 191: A3654.

5 Hoefsloot W, van Ingen J, Andrejak C, et al. The geographic diversity of nontuberculous mycobacteria isolated from pulmonary samples: an NTM-NET collaborative study. Eur Respir J 2013; 42: 1604-1613.

6 Redding GJ, Singleton RJ, Valery PC, et al. Respiratory exacerbations in indigenous children from two countries with non-cystic fibrosis chronic suppurative lung disease/bronchiectasis. Chest 2014; 146: 762-774.

7 Chalmers JD, Aliberti S, Blasi F. Management of bronchiectasis in adults. Eur Respir J 2015; 45: 1446-1462.

8 Chalmers JD, Aliberti S, Polverino E, et al. The EMBARC European Bronchiectasis Registry: protocol for an international observational study. ERJ Open Res 2016; 2: 00081-2015.

9 Aliberti S, Hill AT, Mantero M, et al. Quality standards for the management of bronchiectasis in Italy: a national audit. Eur Respir J 2016; 48: 244-248. 